\title{
Efficacy of first-line erlotinib in non-small cell lung cancer patients undergoing dose reduction and those with a low body surface area: A population-based observational study by the Ibaraki Thoracic Integrative (POSITIVE) Research Group
}

\author{
MASAHARU INAGAKI ${ }^{1}$, YOKO SHINOHARA ${ }^{1}$, TAKAYUKI KABURAGI $^{2}$, SHINSUKE HOMMA $^{3}$, \\ NOBUYUKI HIZAWA $^{3}$, HIROYUKI NAKAMURA ${ }^{4}$, KENJI HAYASHIHARA ${ }^{5}$, TAKEFUMI SAITO ${ }^{5}$, \\ HIROICHI ISHIKAWA ${ }^{6}$, HIDEO ICHIMURA ${ }^{7}$, TAKESHI NAWA ${ }^{7}$, NORIHIRO KIKUCHI ${ }^{8}$, \\ KUNIHIKO MIYAZAKI ${ }^{9}$, TAKAHIDE KODAMA ${ }^{9}$, KOICHI KAMIYAMA ${ }^{10}$, \\ HIROAKI SATOH $^{11}$ and KINYA FURUKAWA ${ }^{4}$
}

${ }^{1}$ Divisions of Thoracic Surgery and Respiratory Medicine, Tsuchiura Kyodo General Hospital and Regional Cancer Center, Tsuchiura, Ibaraki 300-0053; ${ }^{2}$ Division of Respiratory Medicine, Ibaraki Prefectural Central Hospital and Regional Cancer Center,

Kasama, Ibaraki 309-1793; ${ }^{3}$ Division of Respiratory Medicine, Faculty of Clinical Medicine, University of Tsukuba,

Tsukuba, Ibaraki 305-8576; ${ }^{4}$ Divisions of Thoracic Surgery and Respiratory Medicine, Tokyo Medical University, Ibaraki Medical Center Hospital, Ami, Ibaraki 300-0395; ${ }^{5}$ Division of Respiratory Medicine, Ibaraki Higashi National Hospital, Tokai, Ibaraki 319-1113; ${ }^{6}$ Division of Respiratory Medicine, Tsukuba Medical Center Hospital and Regional Cancer Center, Tsukuba, Ibaraki 305-8558; ${ }^{7}$ Divisions of Thoracic Surgery and Respiratory Medicine, Hitachi General Hospital, Hitachi, Ibaraki 317-0077; ${ }^{8}$ Division of Respiratory Medicine, Kasumigaura Medical Center, Tsuchiura, Ibaraki 300-0812;

${ }^{9}$ Division of Respiratory Medicine, Ryugasaki Saiseikai General Hospital, Ryugasaki, Ibaraki 301-0854;

${ }^{10}$ Division of Thoracic Surgery, Tsukuba Kinen General Hospital, Tsukuba, Ibaraki 300-2622;

${ }^{11}$ Division of Respiratory Medicine, Mito Medical Center, Institute of Clinical Medicine, University of Tsukuba, Mito, Ibaraki 310-0015, Japan

Received July 9, 2015; Accepted November 23, 2015

DOI: $10.3892 / \mathrm{mco} .2015 .720$

\begin{abstract}
The aim of the present study was to evaluate the efficacy of erlotinib, one of the epidermal growth factor receptor-tyrosine kinase inhibitors (EGFR-TKIs), in patients undergoing dose reduction and in those with a low body surface area (BSA). The association between dose reduction, low BSA and efficacy, including response rate, disease control rate, time to treatment failure and overall survival, were evaluated in patients prescribed first-line erlotinib for EGFR mutated non-small cell lung cancer patients between April 2012 and March 2015. A total of 22 patients received first-line erlotinib during the study period. A dose reduction of erlotinib for the reason of low BSA and poor performance status occurred in $14(63.6 \%)$ of the
\end{abstract}

Correspondence to: Professor Hiroaki Satoh, Division of Respiratory Medicine, Mito Medical Center, Institute of Clinical Medicine, University of Tsukuba, Miya-machi 3-2-7, Mito, Ibaraki 310-0015, Japan

E-mail: hirosato@md.tsukuba.ac.jp

Key words: erlotinib, dose reduction, survival, first-line, retrospective, population-based patients: $6(27.3 \%)$ had initial dose reduction, $6(27.3 \%)$ had dose reduction in their clinical courses, and $2(9.1 \%)$ had both. Dose reduction of erlotinib with the initial dose of erlotinib/BSA was $>80 \mathrm{mg} / \mathrm{m}^{2}$, and longest-term prescribed dose of erlotinib/BSA was $>50 \mathrm{mg} / \mathrm{m}^{2}$, which may have no association with a survival disadvantage. Dose-reduction estimation studies for TKIs may be crucial, particularly for patients with a low BSA. Future prospective studies and confirmation of these results in population-based retrospective ones investigating the incidence of dose reduction in patients with AEs and those with low BSA may be required for the efficient use of erlotinib in common clinical practice.

\section{Introduction}

Determining the recommended dose of traditional cytotoxic antitumor agents usually occurs at or near their maximum-tolerated dose (1-3). The recommended dose for epidermal growth factor receptor-tyrosine kinase inhibitors (EGFR-TKIs) is established at an optimal biological dose, which minimizes the risk of adverse events (AEs) without compromising efficacy (4-6). However, previous dose-finding studies did not factor in body size or body surface area (BSA). A significant portion of EGFR-mutated patients are female 
and the majority may have low BSA (7-9). Therefore, these patients may require a dose reduction in daily clinical practice due to AEs or low BSA. However, limited information is available regarding the association between low BSA and TKI dose reduction and efficacy $(10,11)$. Furthermore, the efficacy of TKIs has not been elucidated in patients undergoing a dose reduction due to severe AEs. In the present study, the efficacy of TKIs was evaluated in patients undergoing a dose reduction due to AEs and in those with a low BSA in clinical practice.

\section{Patients and methods}

Patients. The Ibaraki Prefecture in Japan covers an area of $6,095 \mathrm{~km}^{2}$ and has a population of 3 million. This retrospective population-based study included patients with non-small cell lung cancer (NSCLC) who had received erlotinib at 16 hospitals in Ibaraki between November 2009 and August 2011. All the patients were required to have had a pathological or cytological diagnosis of NSCLC. Pathological diagnosis of lung cancer was defined by the World Health Organization classification (12). A tumor-node-metastasis staging procedure using head computed tomography (CT) or magnetic resonance imaging, bone scans, and ultrasonography and/or CT of the abdomen was performed for all patients prior to starting erlotinib treatment. Eligible patients were identified in the clinical database of each hospital and the following information was extracted from their data: Patient demographics at the time of erlotinib treatment (age, gender, smoking history, histology and stage) and objective tumor response. The tumor response was evaluated according to the Response Evaluation Criteria in Solid Tumors (RECIST). Toxicity was graded according to the National Cancer Institute Common Toxicity Criteria, version 3.0 (13).

Evaluation of outcomes. Response rate, time to treatment failure (TTF) and overall survival (OS) after the initiation of erlotinib therapy in each treatment group with or without EGFR mutation were evaluated.

Ethical statement. This observational study conformed to the Ethical Guidelines for Clinical Studies issued by the Ministry of Health, Labor and Welfare of Japan. Ethical approval of reporting this study was obtained from the institutional review board in the Mito Medical Center, University of Tsukuba (Mito, Ibaraki, Japan) (13-15).

Statistical analysis. Differences in proportions between 2 independent groups were compared by the $\chi^{2}$ test. $\mathrm{P}<0.05$ was considered to indicate a statistically significant difference. Survival probability was estimated with the Kaplan-Meier method and compared using the log-rank test. In multivariate analysis, Cox's proportional model was used.

\section{Results}

Patient characteristics. The patient characteristics are summarized in Table I. During the study period, a total of 22 patients were treated with first-line erlotinib. The median age was 72 years (range, 33-89 years). A total of 4 patients $(18.2 \%)$ were male, $3(13.6 \%)$ had a smoking history and $14(63.6 \%)$ had a good performance status (0-1). All the patients had
Table I. Characteristics of the 22 patients with EGFR-mutated non-small cell lung cancer.

\begin{tabular}{lc}
\hline Characteristics & Patients \\
\hline Median age, years (range) & $72(33-89)$ \\
Gender, $\mathrm{n}$ & \\
Male & 4 \\
Female & 18 \\
Smoking history, $\mathrm{n}$ & \\
Never smokers & 19 \\
Current or ex-smoker & 3 \\
ECOG PS, $\mathrm{n}$ & \\
$0-1$ & 14 \\
$2-4$ & 8 \\
Histology, $\mathrm{n}$ & \\
Adenocarcinoma & 22 \\
Squamous & 0 \\
Large cell & 0 \\
Other & 0 \\
EGFR mutation, $\mathrm{n}$ & \\
Ex 19 del & 13 \\
Ex 21 L858R & 15 \\
Body surface area, $\mathrm{n}$ & 7 \\
$<1.25 \mathrm{~m}^{2}$ & \\
$\geq 1.25$ to $<1.50 \mathrm{~m}^{2}$ & \\
$\geq 1.50 \mathrm{~m}^{2}$ & \\
& \\
& \\
&
\end{tabular}

EGFR, epidermal growth factor receptor; ECOG PS, Eastern Cooperative Oncology Group performance status.

adenocarcinoma. A total of 15 patients had Ex19 del, and 7 had Ex 21 L858R. One patient (4.5\%) had an extremely low BSA $\left(<1.25 \mathrm{~m}^{2}\right)$ and $13(59.1 \%)$ had $1.25<$ BSA $<1.5 \mathrm{~m}^{2}$. A dose reduction of erlotinib for the reason of low BSA and poor PS was performed in $14(63.6 \%)$ of the patients: $6(27.3 \%)$ had initial dose reduction, $6(27.3 \%)$ had dose reduction in their clinical courses, and 2 (9.1\%) had both.

Overall response rate $(R R)$, disease control rate $(D C R)$, progression-free survival (PFS) and AEs. The overall RR was $77.3 \%$, the DCR was $100 \%$ and the TTF was 13.0 months, and OS was did not reach the median survival time (range, 1-21 months). Among 14 patients with low BSA $\left(<1.5 \mathrm{~m}^{2}\right), 11(78.6 \%)$ had dose reduction and $10(71.4 \%)$ had PR (at least a $30 \%$ decrease in the sum of the largest diameter of target lesions, taking as reference the baseline sum largest diameter in evaluation of target lesions in RECIST criteria). By contrast, 3 (37.5\%) of 8 patients with BSA $>1.5$ had dose reduction and $7(87.5 \%)$ had $\mathrm{PR}$. There was no statistical difference in dose reduction and response between them $(\mathrm{P}=0.0541$ and $\mathrm{P}=0.3869$, respectively). Among 14 patients with low BSA $\left(<1.5 \mathrm{~m}^{2}\right), 5(35.7 \%)$ had grade 3-4 AEs, and $3(37.5 \%)$ of 8 patients with $\mathrm{BSA} \geq 1.5 \mathrm{~m}^{2}$ had grade $3-4$ AEs. There was no statistical difference in AEs between them $(\mathrm{P}=0.9332)$. 
Table II. Dose reduction of erlotinib.

\begin{tabular}{lc}
\hline Erlotinib dose & Patients \\
\hline Dose reduction & $14(63.6)$ \\
Present, $\mathrm{n}(\%)$ & 6 \\
$\quad$ Initial dose reduction, $\mathrm{n}$ & 6 \\
$\quad$ Dose reduction in clinical course, & 2 \\
Both, $\mathrm{n}$ & $8(36.4)$ \\
Absent, n (\%) & $86.2(67.6-121)$ \\
Median initial dose/BSA, mg/m²/day (range) & \\
Median longest-term prescribed dose/BSA, & $74.1(28.7-121)$ \\
mg/m²/day (range) & \\
\hline BSA, body surface area. & \\
\hline
\end{tabular}

Table III. Dose reduction of erlotinib and survival difference.

\begin{tabular}{lcccc}
\hline & & & \multicolumn{2}{c}{$\begin{array}{c}\text { Log-rank test } \\
\text { P-value }\end{array}$} \\
\cline { 4 - 5 } $\begin{array}{l}\text { Dose, } \\
\text { mg/m²/BSA level }\end{array}$ & $\begin{array}{c}\text { <Cut-off } \\
\text { level, } n\end{array}$ & $\begin{array}{c}\text { CCut-off } \\
\text { level, } n\end{array}$ & TTF & OS \\
\hline Cut-off initial & & & & \\
80 & 8 & 14 & 0.9920 & 0.7453 \\
90 & 13 & 9 & 0.6649 & $\mathrm{CA}$ \\
100 & 15 & 7 & 0.8077 & $\mathrm{CA}$ \\
110 & 18 & 4 & 0.3668 & $\mathrm{CA}$ \\
Longest-term & & & & \\
50 & 3 & 19 & 0.3349 & $\mathrm{CA}$ \\
60 & 4 & 18 & 0.7433 & $\mathrm{CA}$ \\
70 & 7 & 15 & 0.9744 & $\mathrm{CA}$ \\
80 & 13 & 9 & 0.3864 & 0.0614 \\
90 & 16 & 6 & $\mathrm{CA}$ & $\mathrm{CA}$ \\
100 & 17 & 5 & $\mathrm{CA}$ & $\mathrm{CA}$ \\
110 & 20 & 2 & $\mathrm{CA}$ & $\mathrm{CA}$ \\
\hline
\end{tabular}

BSA, body surface area; CA, cannot analyze as OS did not reach the median survival time; TTF, time to treatment failure; OS, overall survival.

Dose reduction and survival. Table II shows the results of dose reduction. TTF in patients with an initial dose of $100 \mathrm{mg}$ erlotinib and those with $150 \mathrm{mg}$ was 9.0 and 9.0 months, respectively, and there was no statistical difference in TTF between them $(\mathrm{P}=0.9920)$. OS in patients with an initial dose of $100 \mathrm{mg}$ erlotinib and those with $150 \mathrm{mg}$ did not reach the median survival time, respectively, and there was no statistical difference in TTF between them $(\mathrm{P}=0.7456)$. With regard to the longest-term prescribed dose (LTD) of erlotinib, TTF in patients administered $\leq 100 \mathrm{mg}$ and those with $>100 \mathrm{mg}$ was 9.0 and 13.0 months, respectively; however, there was no statistical difference in TTF between them $(\mathrm{P}=0.3684)$. OS in these patients did not reach the median survival time, respectively, and there was no statistical difference in TTF $(\mathrm{P}=0.0614)$.
Dose reduction, BSA and survival. Dose reduction and survival were evaluated with consideration of BSA. Patients were divided into groups based on whether their initial erlotinib dose (ID)/BSA levels were $<$ or $>80 \mathrm{mg} / \mathrm{m}^{2}$ and subdivided into groups with ID/BSA levels of 90,100 and $110 \mathrm{mg} / \mathrm{m}^{2}$. As shown in Table III, a statistically significant difference was not observed when patients were divided into groups at ID/BSA levels of $80,90,100$ and $110 \mathrm{mg} / \mathrm{m}^{2}$. Patients were divided into groups based on whether their LTD/BSA levels were $<$ or $>50 \mathrm{mg} / \mathrm{m}^{2}$ and subdivided into groups with LTD/BSA levels of $60,70,80,90,100$ and $110 \mathrm{mg} / \mathrm{m}^{2}$. As shown in Table III, a statistically significant difference was not observed.

\section{Discussion}

A fixed dose of EGFR-TKIs achieved a significant improvement in PFS with acceptable AE profiles in previous clinical trials $(14,15)$. The first-choice treatments for advanced NSCLC patients with EGFR mutation include gefitinib, erlotinib or afatinib (14,15). However, TKI efficacy in the clinical setting, particularly in patients undergoing a dose reduction due to toxicity or low BSA, remains to be elucidated. Whether a strictly fixed dose of TKIs, as determined by dose-finding studies, would exhibit the same efficacy in patients undergoing a dose reduction as in those without also remains to be elucidated. Therefore, the RR, DCR, PFS and OS in patients with and those without a dose reduction of TKIs were determined in our previous study (11). However, the study included heterogeneity in the EGFR mutation status, TKIs used, and second or later line of TKI treatment. To eliminate the effect on the results of unknown factors associated with them, the present study was performed using a database of patients treated with first-line erlotinib. Dose reduction of erlotinib for the reason of low BSA and poor PS occurred in $14(63.6 \%)$ of the patients: $6(27.3 \%)$ had initial dose reduction, $6(27.3 \%)$ had dose reduction in their clinical courses, and $2(9.1 \%)$ exhibited both. In previous studies assessing TKI treatments, either in clinical trials or clinical practice, the population of female patients with NSCLC was higher compared to that in the general population (7-9,16). The present study identified no disadvantage regarding TTF from a dose reduction in patients receiving first-line erlotinib, even when considering BSA. Although these findings were obtained from a small number of patients in a retrospective study, no definitive conclusions could be drawn on how dose reduction would affect patients with AEs and those with low BSA. Therefore, these results be should be interpreted carefully, rather than overlooking them as anecdotal findings. The most appropriate dosage of erlotinib for such patients should possibly be recalculated, in order to obtain maximum efficacy with an acceptable toxicity profile.

To the best of our knowledge, only two previous studies have evaluated the effect of BSA on the efficacy of TKIs; the study by Ichihara et al (10) and our previous study (11). The former study investigated the potential effect of BSA on the PFS and OS of patients with advanced EGFR-mutated NSCLC who were treated with gefitinib (10) and identified that BSA affected the outcome of gefitinib therapy, with a higher BSA being associated with worse PFS (10). The main focus of the study by Ichihara et al (10) was treatment efficacy in patients with a high BSA. By contrast, the present study aimed to assess the effects of dose reduction in patients with AEs, as 
well as in patients with a low BSA. The PFS of patients with low BSA with treatment interruption or reduced dose was either equal to or superior to that of all low-BSA patients (10), consistent with our previous study (11) and the present results. Ichihara et al (10) hypothesized that dose reduction in patients due to AEs may be those with relatively high blood concentrations of the agent, due to factors including drug metabolism. This hypothesis requires confirmation in future studies.

The effect of BSA on OS was also investigated. There was no association between OS and BSA in the study by Ichihara et al (10). In our previous study, there was no apparent difference in OS between patients with BSA $<1.5 \mathrm{~m}^{2}$ and those $>1.5 \mathrm{~m}^{2}$ receiving treatment with either gefitinib or erlotinib (11). The OS in patients without dose reduction appeared to be longer compared to patients with dose reduction, with no statistical significance (11). Ichihara et al (10) suggested that this was possibly the effect of preceding and/or subsequent therapies on OS, and $>70 \%$ of the patients received chemotherapy following disease progression post-gefitinib monotherapy, therefore, post-progression therapy may have hindered any difference in PFS between the high- and low-BSA subgroups. Further cytotoxic antitumor chemotherapy was not indicated in the majority of the low-BSA patients, as observed in our previous study, which may also be an explanation (11). In the present study, taking BSA into consideration, there was no significant disadvantage in the ID level and LTD level for patients with low BSA. These results suggested that the degree of dose reduction conducted in daily practice for low BSA, poor PS and severe AEs has little influence on the survival rate.

There are certain limiting factors for the analysis of the present data set: Small sample size, retrospective design, reasons for dose reduction, and exclusion of patients with discontinuation of erlotinib without any dose reduction. Another major limitation was that pharmacokinetic data were not included in this analysis. Whether BSA differences cause inter-patient pharmacokinetic variability, resulting in the observed difference in TTF remains to be elucidated, possibly by a pharmacokinetics-pharmacodynamics study. The blood concentration of cytotoxic agents is closely associated with their efficacy (17-19), and similarly, the blood concentration of TKIs appears to be associated with their efficacy $(20,21)$.

The present results suggested that dose reduction of erlotinib with ID/BSA $>80 \mathrm{mg} / \mathrm{m}^{2}$, and LTD/BSA $>50 \mathrm{mg} / \mathrm{m}^{2}$ may not be associated with survival disadvantage. Dose-reduction estimation studies for TKIs may be crucial, particularly for low-BSA patients. Heterogeneity in these factors, such as low BSA, poor PS and severe AEs, should be taken into consideration. Prospective studies, followed by confirmation in retrospective ones, which investigate the incidence of dose reduction in patients with AEs and those with low BSA may be important for common clinical practice. This approach may further elucidate the clinical meaning of TKI dose reduction in such patients.

\section{References}

1. Seymour L and Eisenhauer E: A review of dose-limiting events in phase I trials: Antimetabolites show unpredictable relationships between dose and toxicity. Cancer Chemother Pharmacol 47: 2-10, 2001.

2. Hoekstra R, Verweij J and Eskens FA: Clinical trial design for target specific anticancer agents. Invest New Drugs 21: 243-250, 2003.
3. Zohar S, Lian Q, Levy V, Cheung K, Ivanova A and Chevret S: Quality assessment of phase I dose-finding cancer trials: Proposal of a checklist. Clin Trials 5: 478-485, 2008.

4. Wolf M, Swaisland $\mathrm{H}$ and Averbuch S: Development of the novel biologically targeted anticancer agent gefitinib: Determining the optimum dose for clinical efficacy. Clin Cancer Res 10: 4607-4613, 2004.

5. Takimoto CH: Maximum tolerated dose: Clinical endpoint for a bygone era? Target Oncol 4: 143-147, 2009.

6. Yamamoto N, Horiike A, Fujisaka Y, Murakami H, Shimoyama T, Yamada Y and Tamura T: Phase I dose-finding and pharmacokinetic study of the oral epidermal growth factor receptor tyrosine kinase inhibitor Ro50-8231 (erlotinib) in Japanese patients with solid tumors. Cancer Chemother Pharmacol 61: 489-496, 2008.

7. Boyer M, Horwood K, Pavlakis N, De Souza P, Millward M, Stein B, Johnston M, Abell F and Rischin D: Efficacy of erlotinib in patients with advanced non-small-cell lung cancer (NSCLC): Analysis of the Australian subpopulation of the TRUST study. Asia Pac J Clin Oncol 8: 248-254, 2012.

8. Gahr S, Stoehr R, Geissinger E, Ficker JH, Brueckl WM, Gschwendtner A, Gattenloehner S, Fuchs FS, Schulz C, Rieker RJ, et al: EGFR mutational status in a large series of Caucasian European NSCLC patients: Data from daily practice. Br J Cancer 109: 1821-1828, 2013.

9. Kaburagi T, Satoh H, Hayashihara K, Endo T, Hizawa N, Kurishima K, Nishimura Y, Hashimoto T, Nakamura H, Kishi K, et al: Observational study on the efficacy and safety of erlotinib in patients with non-small cell lung cancer. Oncol Lett 5: 435-439, 2013

10. Ichihara E, Hotta K, Takigawa N, Kudo K, Kato Y, Honda Y, Hayakawa H, Minami D, Sato A, Tabata M, et al: Impact of physical size on gefitinib efficacy in patients with non-small cell lung cancer harboring EGFR mutations. Lung Cancer 81: 435-439, 2013.

11. Sato S, Kurishima K, Miyazaki K, Kodama T, Ishikawa H, Kagohashi K, Tamura T, Homma S, Satoh H and Hizawa N: Efficacy of tyrosine kinase inhibitors in non-small-cell lung cancer patients undergoing dose reduction and those with a low body surface area. Mol Clin Oncol 2: 604-608, 2014.

12. Travis WD, Brambilla E, Muller-Hermelink HK, Harris CC (eds): Pathology and Genetics: Tumours of the Lung, Pleura, Thymus and Heart. IARC Press, Lyon, 2004.

13. Cancer Therapy Evaluation Program, Common Toxicity Criteria for Adverse Events, Version 3.0. http://ctep.cancer.gov. Accessed May 15, 2014.

14. Pennell NA: Integration of EGFR inhibitors and conventional chemotherapy in the treatment of non-small-cell lung cancer. Clin Lung Cancer 12: 350-359, 2011.

15. Roengvoraphoj M, Tsongalis GJ, Dragnev KH and Rigas JR: Epidermal growth factor receptor tyrosine kinase inhibitors as initial therapy for non-small cell lung cancer: Focus on epidermal growth factor receptor mutation testing and mutation-positive patients. Cancer Treat Rev 39: 839-850, 2013.

16. Hayashibara K, Satoh H, Shinohara Y, Inagaki M, Kaburagi T, Hashimoto T, Kurishima K, Ishikawa $\mathrm{H}$, Ichimura $\mathrm{H}$, Nawa T, et al: A population-based study of gefitinib in patients with non-small cell lung cancer. Med Oncol 26: 222-227, 2009.

17. Miles DW, Chan A, Dirix LY, Cortés J, Pivot X, Tomczak P, Delozier T, Sohn JH, Provencher L, Puglisi F, et al: Phase III study of bevacizumab plus docetaxel compared with placebo plus docetaxel for the first-line treatment of human epidermal growth factor receptor 2-negative metastatic breast cancer. J Clin Oncol 28: 3239-3247, 2010.

18. Wittenburg LA and Gustafson DL: Optimizing preclinical study design in oncology research. Chem Biol Interact 190: 73-78, 2011.

19. Fujita KI and Sasaki Y: Optimization of cancer chemotherapy on the basis of pharmacokinetics and pharmacodynamics: From patients enrolled in 'clinical trials' to those in the 'real world'. Drug Metab Pharmacokinet 29: 20-28, 2014.

20. Fukudo M, Ikemi Y, Togashi Y, Masago K, Kim YH, Mio T, Terada $\mathrm{T}$, Teramukai $\mathrm{S}$, Mishima $\mathrm{M}$, Inui $\mathrm{K}$ and Katsura $\mathrm{T}$ : Population pharmacokinetics/pharmacodynamics of erlotinib and pharmacogenomic analysis of plasma and cerebrospinal fluid drug concentrations in Japanese patients with non-small cell lung cancer. Clin Pharmacokinet 52: 593-609, 2013.

21. Ranson M and Wardell S: Gefitinib, a novel, orally administered agent for the treatment of cancer. J Clin Pharm Ther 29: 95-103, 2004. 